\title{
Uropathogenic $E$. coli infection provokes epigenetic downregulation of CDKN2A (p16INK4A) in uroepithelial cells
}

\author{
Cornelia Tolg ${ }^{1}$, Nesrin Sabha ${ }^{1}$, Rene Cortese ${ }^{2}$, Trupti Panchal ${ }^{1}$, Alya Ahsan ${ }^{1}$, Ashraf Soliman ${ }^{1}$, Karen J Aitken ${ }^{1}$, \\ Arturas Petronis ${ }^{2}$ and Darius J Bägli ${ }^{1}$
}

Host cell and bacterial factors determine severity and duration of infections. To allow for bacteria pathogenicity and persistence, bacteria have developed mechanisms that modify expression of host genes involved in cell cycle progression, apoptosis, differentiation and the immune response. Recently, Helicobacter pylori infection of the stomach has been correlated with epigenetic changes in the host genome. To identify epigenetic changes during Escherichia coli induced urinary tract infection (UTI), we developed an in vitro model of persistent infection of human uroepithelial cells with uropathogenic E. coli (UPEC), resulting in intracellular bacteria colonies. Cells inoculated with FimH-negative E. coli (N-UPEC) that are not internalized and non-inoculated cells were used as controls. UPEC infection significantly induced de novo methyltransferase (DNMT) activity (12.5-fold $P=0.002$ UPEC vs non-inoculated and 250 -fold $P=0.001$ UPEC vs N-UPEC inoculated cells) and Dnmt 1 RNA expression (6-fold $P=0.04$ UPEC vs non-inoculated cells) compared with controls. DNMT1 protein levels were significantly increased in three uroepithelial cell lines (5637, J82, HT-1197) in response to UPEC infection as demonstrated by confocal analysis. Real-time PCR analysis of candidate genes previously associated with bacteria infection and/or innate immunity, revealed UPEC-induced downregulation of the tumor suppressor gene CDKN2A (3.3-fold $P=0.007$ UPEC vs non-inoculated and 3.3-fold $P=0.001$ UPEC vs N-UPEC) and the DNA repair gene MGMT (9-fold $P=0.03$ UPEC vs non-inoculated). Expression of CDH1, MLH1, DAPK1 and TLR4 was not affected. Pyrosequencing of CDKN2A and MGMT CpG islands revealed increased methylation in CDKN2A exon 1 (3.8-fold $P=0.04$ UPEC vs N-UPEC and UPEC vs non-inoculated). Methylation of MGMT was not affected. UPEC-induced methylation of CDKN2A exon 1 may increase bladder cancer and presage UTI risk, and be useful as a biological marker for UTI susceptibility or recurrence.

Laboratory Investigation (2011) 91, 825-836; doi:10.1038/labinvest.2010.197; published online 17 January 2011

KEYWORDS: CDKN2A; DNA methylation; epigenetics; urinary tract infection; uropathogenic $E$. coli; urothelium

Urinary tract infection (UTI) results in significant morbidity and accounts for about 8 million doctor visits each year in the United States alone. ${ }^{1,2}$ Although UTI can be treated with antibiotics, a significant number of patients are prone to recurrent UTI. Recurrent UTI, which is often resistant to antibiotic therapy, causes medical challenges particularly in pediatric or immunocompromised patients. For example, urinary tract abnormalities such as vesicoureteral reflux or a dilated upper urinary tract often increase UTI susceptibility. Even without aggravating anatomic risk factors, it is not known whether the intrinsic biologic propensity to develop a recurrent UTI differs between patients in the general population. The symptoms of UTI are wide ranging and include pain, fever, incontinence, aberrant voiding patterns often inducing chronic bladder dysfunction, renal parenchymal damage, nephron loss and renovascular hypertension. Morbidity, multisystem complications and sepsis requiring hospitalization for febrile UTI commonly burden the provider system. Co-existing immunocompromised states (chemotherapy, transplantation, HIV) can be life threatening, with UTI in some cases precipitating mortality. Furthermore, although still controversial, several studies

\footnotetext{
${ }^{1}$ Division of Urology, Developmental and Stem Cell Biology, Hospital for Sick Children, Research Institute, Institute of Medical Sciences, University of Toronto, Toronto, ON, Canada and ${ }^{2}$ The Krembil Family Epigenetics Laboratory CAMH—Centre for Addiction and Mental Health, Toronto, ON, Canada

Correspondence: Dr DJ Bägli, MDCM, Division of Urology and Developmental and Stem Cell Biology, The Hospital for Sick Children, Research Institute, 555 University Avenue M292, Toronto, ON, M5G 1X8 Canada.

E-mail: darius.bagli@sickkids.ca

Received 5 December 2009; revised 14 October 2010; accepted 15 November 2010
} 
identified a correlation between recurrent UTI and bladder cancer risk. ${ }^{3,4}$

Escherichia coli, belonging to a family of gram-negative bacilli, Enterobacteriaceae, accounts for $>80 \%$ of acute UTI in children. ${ }^{5}$ Uroepithelial cells lining the entire urinary tract are the first cells of contact for uropathogenic E. coli (UPEC). Clinical UTI results from a cascade of events beginning with binding of adherence factors (e.g. FimH) that coat the UPEC surface to uroplakins on the uroepithelial cells surface. ${ }^{6}$ This binding results in UPEC invasion into the uroepithelial cells. ${ }^{7}$ Internalized UPEC form intracellular bacterial communities, which are self-contained, actively multiplying clusters of UPEC that reside within the cytoplasm of uroepithelial cells. Acute UPEC infection stimulates uroepithelial cell apoptosis resulting in release of intracellular bacteria and infection of adjacent cells. After the initial stages of active infection, a few UPEC escape the host immune response, become dormant and form quiescent intracellular bacteria reservoirs (QIR), which can persist for months inside uroepithelial cells. ${ }^{8}$ Reactivation of the QIR may lead to recurrence of UTI. ${ }^{8}$ Although the mechanism of QIR maintenance and reactivation has not been clearly delineated, it is likely that UTI responsive cellular mechanisms within the uroepithelial cells are co-opted to have a role.

Susceptibility to UTI, as with other infections, is generally influenced by host factors in response to specific bacterial virulence factors and involves a repertoire of different adherence factors, including hyaluronan, CD44 and uroplakins, which bind the FimH adhesin molecules of the pathogenic bacteria. ${ }^{9}$ The host cell response to UTI involves activation of an innate immune response, apoptosis, increased proliferation and differentiation, implicating activation of cell surface receptors such as the Toll-like receptors (TLR) leading to transcription of pro-inflammatory cytokines, such as interleukin-6 (IL6) and interleukin-8 (IL8), ultimately recruiting neutrophils to the site of infection. Recruitment of neutrophils and increased uroepithelial cell apoptosis results in exfoliation of infected uroepithelial cells into the urine thereby decreasing bacteria burden and the risk for reinfection. ${ }^{10-13}$

While UTI is clearly multifactorial in nature, ${ }^{14}$ genetic variability in the host may underlie some forms of susceptibility for UTI. ${ }^{15-17}$ For example, mutations and polymorphisms in members of the TLR family that recognize pathogen-associated molecular patterns can predispose to UTI in mice, ${ }^{18,19}$ and humans. ${ }^{20,21}$ Nevertheless, while UTI susceptibility may be influenced by some inherited predisposition, it remains largely sporadic and is most likely a nonMendelian trait.

Epigenetics is the study of long-term changes in gene function which persist through many cell divisions without changes in the DNA sequence. ${ }^{22}$ Epigenetic mechanisms in eukaryotes include DNA methylation, histone modification and expression of small non-coding microRNA sequences, all of which contribute to gene silencing. Since methylation and histone modifications are relatively simple chemical alterations extrinsic to the DNA sequence, they are often viewed as one means by which external or environmental factors bring about differential expression for a given DNA sequence. DNA methylation is the addition of a methyl group to the carbon position 5 on cytosine rings in $\mathrm{CpG}$ dinucleotides. It has an important role in development, differentiation, chromatin structure, transcriptional regulation and genomic stability in normal cells. Thus far, three de novo methyltransferases (DNMTs), DNMT1, DNMT3a and DNMT3b, have been identified in mammalian cells. DNMT1 is the most critical for controlling the methylation state of DNA in somatic cells. $^{22}$ Increased DNA methylation (hypermethylation) is often associated with loss of gene expression, although this paradigm has recently been challenged by the observation that expression of a significant number of genes is downregulated in Dnmt-deficient cells. Nevertheless, methylationassociated downregulation of genes can be seen in host cells in response to viral, parasitic and bacterial infections, including Schistosoma infection of the bladder ${ }^{23}$ and Helicobacter pylori or Epstein-Barr virus infection of the stomach. ${ }^{24-27}$ Human papillomavirus E7 and adenovirus E1A proteins have also been shown to directly associate with DNMT1 and stimulate DNMT1 activity. ${ }^{28}$ Induction of latent membrane protein 1 of Epstein-Barr virus has been shown to directly activate DNMT1 expression in cultured cancer cells ${ }^{29}$ as does Hepatitis B viral infection of a human hepatocellular carcinoma cell line. ${ }^{30}$

In addition to genetic alterations, epigenetic silencing by methylation of the promoter region is well documented for many growth-suppressor genes, such as cyclin-dependent kinase inhibitor 2A (CDKN2A (P16INK4A)), mutL homolog 1 (MLH1), O6-methylguanine-DNA-methyltransferase (MGMT) and deathassociated protein kinase 1 (DAPK1) resulting in 'suppressing the suppressors. ${ }^{31,32}$ P16INK4A initiates cell cycle arrest by inhibiting cyclin-dependent kinase 4 (CDK4) during induced cell stress, for example, by chronic infection. Indeed, methylation-mediated silencing of several of these genes has been implicated in Schistosoma-associated bladder carcinoma ${ }^{23}$ and in gastric H. pylori infection mentioned above. ${ }^{24-26}$

Promoter methylation is also a common mechanism for downregulation of MLH1 and MGMT, two members of the DNA repair system. ${ }^{33}$ This system has a major role in maintaining genomic stability and integrity by correcting errors during DNA replication. Defects in the DNA repair system are associated with accumulation of potential mutations or a 'mutator phenotype' especially in the microsatellite regions of DNA. Loss of this system predisposes to unregulated growth. Loss of MGMT expression increases the numbers of genetic mutations resulting from incorrect DNA replication ${ }^{34}$ thereby contributing to neoplastic progression.

Given the described correlation between DNA methylation and disease progression, we hypothesized that the environmental stress of UTI may lead to modification of the uroepithelial cell epigenome and transcriptome thereby increasing 
susceptibility to UTI recurrence and possibly bladder carcinoma. Here, we describe the use of an in vitro UTI model to study epigenetic consequences of UTI and show by proof-ofprinciple a correlation between promoter methylation and loss of gene expression in infected uroepithelial cells.

\section{MATERIALS AND METHODS}

\section{Bacteria Strains and Cell Lines}

The E. coli strains UT189 and SLC2-35-1 were generously provided by Dr Scott Hultgren (Washington University, St Louis, MO, USA). SLC2-35-1 bacteria lack the FimH adhesion and are unable to invade host cells. The HT-1197, J82 and 5637 cell lines were obtained from ATCC. These cell lines were isolated from human uroepithelial carcinoma and are commonly used to induce UPEC infections. ${ }^{9}$

\section{Infection}

Cells were cultured in either RPMI-1640 + 10\% FCS (5637) or EMEM $+10 \%$ FCS (HT-1197, SLC-35-1) at $37^{\circ} \mathrm{C}, 5 \%$ $\mathrm{CO}_{2}$ and humidified atmosphere. In all, $80 \%$ confluent cultures were inoculated with two E. coli strains: UPEC (UT189, FimH $[+]$ ) and N-UPEC (SLC-35-1, Fim $\mathrm{H}[-]$ ) at the optimized multiplicity of infection (MOI) of 2.5-5 E. coli per cell, for $2 \mathrm{~h}$. Post-inoculation, cells were washed with PBS + Gentamycin $(100 \mu \mathrm{g} / \mathrm{ml})$ followed by incubation in Gentamycin $(10 \mu \mathrm{g} / \mathrm{ml})$ containing medium (EMEM, 10\% FBS) for up to 6 days. Cells that were not inoculated with bacteria were used as negative control. Cells exposed to N-UPEC (SLC-35-1) were used to control for any non-Fim $\mathrm{H}$-related responses to E. coli infection. The term inoculation' refers to exposure of cells to UPEC or N-UPEC bacteria. The term 'infection' refers to uptake of UPEC by host cells.

\section{Immunofluorescence and Image Analysis}

Cells were cultured and inoculated in chamber slides. Cells were fixed in $4 \%$ paraformaldehyde/PBS pH 7.4 for $10 \mathrm{~min}$ at room temperature followed by a 5-min wash step with PBS. Incubation with $3 \% \mathrm{BSA} / \mathrm{PBS}$ for $1 \mathrm{~h}$ at room temperature was used to block non-specific binding. Anti-DNMT1 and E. coli antibodies (Abcam, Cambridge, MA, USA) were used at a $1: 100$ dilution in $1 \%$ BSA/PBS and slides were incubated at $4{ }^{\circ} \mathrm{C}$ overnight. Incubation with non-immune $\operatorname{IgG}$ was used as negative control. Following antibody incubation, slides were washed three times for 5 min with PBS at room temperature. FITC- or TRITC-labeled secondary antibodies (Jackson ImmunoResearch Laboratories, West Grove, PA, USA) were used at a 1:200 dilution in 1\% BSA/PBS and slides were incubated for $30 \mathrm{~min}$ at room temperature. Following secondary antibody incubation, slides were washed three times for $5 \mathrm{~min}$ with PBS. Hoechst dye was used as nuclear stain at a 1:2000 dilution and slides were incubated for $10 \mathrm{~min}$ at room temperature. Following a 5-min wash step with PBS, slides were mounted with fluorescence mounting medium (Dako, Burlington, Canada). Images were obtained using an inverted confocal microscope (Carl Zeiss
Microimaging, Thornwood, NY, USA) and LSM software. Fluorescence was quantified with ImageJ software (http:// rsbweb.nih.gov/ij/).

\section{Quantification of DNMT Activity}

In all, 5637 cells were inoculated as described above and DNMT1 activity was quantified using EpiQuik DNA Methyltransferase Activity kit (Epigentek, Brooklyn, NY, USA) following the manufacturer's guidelines.

\section{RT-PCR}

In all, 5637 cells were inoculated as described above. At 6 days post-inoculation, total RNA was isolated using the RNAeasy kit (Qiagen, Mississauga, ON, USA) following the manufacturer's instructions. cDNA synthesis was performed following the manufacturer's instructions using $0.5 \mu \mathrm{g}$ of total RNA, SuperScript III Reverse Transcriptase and oligo dT (Invitrogen, Carlsbad, CA, USA). This cDNA was used as template for quantitative real-time PCR utilizing the DyNAmo SYBR green qPCR kit (Finnzymes, Woburn, MA, USA). PCR conditions: $94^{\circ} \mathrm{C} 15 \mathrm{~min} ; 45$ cycles of $94^{\circ} \mathrm{C} 30 \mathrm{~s}$; $55^{\circ} \mathrm{C} 30 \mathrm{~s} ; 72^{\circ} \mathrm{C} 30 \mathrm{~s} ; 72^{\circ} \mathrm{C} 5 \mathrm{~min}$. Primer 3 software was used to design forward and reverse primers for each candidate gene (Table 1). Amplification of the housekeeping gene Glyceraldehyde-3-phosphate dehydrogenase $(G A P D H)$ was

\section{Table 1 RT-PCR primers}

\begin{tabular}{llcc}
\hline Gene & Sequence & Tm $\left({ }^{\circ} \mathrm{C}\right)$ & $\begin{array}{c}\text { Product } \\
\text { size (nt) }\end{array}$ \\
\hline CDKN2A forward & ATATGCCTCCCCCACTACC & 56 & 130 \\
CDKN2A reverse & CGTGAGTGCTCACTCCAGAA & & \\
TLR4 forward & TCCATAAAAGCCGAAAGTG & 56 & 119 \\
TLR4 reverse & GATACCAGCACGACTGCTCA & & \\
MGMT forward & GGCACCGCTGTATAAAGGA & 58 & 145 \\
MGMT reverse & ATAGAGCAAGGGCAGCGTA & & \\
MLH1 forward & CAGAGGAAGATGGTCCCAAA & 58 & 116 \\
MLH1 reverse & CAGGTCCCTTCCTCATCAA & & \\
IL8 forward & TAGCCAGGATCCACAAGTCC & 56 & 117 \\
IL8 reverse & GCTTCCACATGTCCTCACAA & & \\
IL6 forward & GAAAGCAGCAAAGAGGCACT & 58 & 108 \\
IL6 reverse & TITCACCAGGCAAGTCTCCT & & \\
DAPK1 forward & ATGATCCCACGTCAATCCAT & 56 & 131 \\
DAPK1 reverse & ACCGAAGGCTATGGGTCT & & \\
E-cadherin forward & AGCTGCCCAGAAATGAAA & 56 & 102 \\
E-cadherin reverse & TTGGCCAGTGATGCTGTAGA & & \\
Dnmt1 forward & ACCAAGCAGGCATCTCTGAC & 56 & 127 \\
Dnmt1 reverse & ACCAGCTTCAGCAGGATGT & & \\
GAPDH forward & GTCAGTGGTGGACCTGACCT & 56 & 147 \\
GAPDH reverse & TGCTGTAGCCAAATTCGTTG & & \\
\hline & & & \\
\hline
\end{tabular}


used as quantitative standard. Real-time PCR reactions were performed on the DNA Engine Opticon 2 Real-Time detection system (Bio-Rad, Hercules, CA, USA).

\section{Pyrosequencing}

Cells were inoculated with UPEC and N-UPEC for $2 \mathrm{~h}$ followed by 6 days post-inoculation incubation as described in the cell culture methods. DNA was isolated using the DNeazy tissue extraction kit (Qiagen) following the manufacturer's instructions. DNA bisulfite conversion and pyrosequencing was performed by EpigenDx (Worcester, MA, USA) using Biotage PyroMark RUO instruments (Biotage, Kungsgatan, Sweden) and primers that were recommended by EpigenDx. The methylation status of each locus was analyzed individually as a T/C SNP using QCpG software (Biotage).

\section{Statistical Analysis}

Student's $t$-test and three-way ANOVA was used for data analysis. $P<0.05$ was considered to be statistically significant.

\section{RESULTS}

\section{UPEC Persist in Infected Uroepithelial Cells In Vitro}

Since we were primarily interested in epigenetic changes resulting from prolonged UPEC infection, we established a chronic UTI in vitro model. In all 5637, J82 and HT-1197 cells, originally isolated from a bladder carcinoma and commonly used as host cells for infection models, ${ }^{35}$ were inoculated with two E. coli strains: UPEC (UT189) and N-UPEC (SLC2-35-1). UPEC adhere and internalize into uroepithelial cells whereas uroepithelial cell binding and internalization of N-UPEC does not occur due to lack of functional FimH fimbrae. ${ }^{36,37}$ First, we optimized the multiplicity of infection (MOI = number of $E$. coli per number of uroepithelial cells), infection time, as well as duration of post-inoculation incubation. Initially, prolonged $(24 \mathrm{~h})$ E. coli infection at MOI of $100 \mathrm{E}$. coli per cell was cytolytic (data not shown). Therefore, we reduced the inoculation period to $2 \mathrm{~h}$ and the MOI to 2.5 to 5 E. coli per cell. To mimic the clinical situation, where patients are treated with antibiotics, as closely as possible, following $2 \mathrm{~h}$ of acute infection, cells were treated with Gentamycin. This treatment removed extracellular (non-internalized) bacteria. Since de novo DNA methylation requires cell proliferation, it was essential that our infection protocol did not interfere with cell proliferation. To compare cell proliferation of UPEC-infected $v s$ noninoculated or N-UPEC inoculated uroepithelial cells, $2 \times 10^{5}$ cells were inoculated (with UPEC or N-UPEC) at a MOI of 5 for $2 \mathrm{~h}$ and cultured post-inoculation in the presence of Gentamycin for up to 6 days. Uroepithelial cell proliferation in response to $E$. coli infection was quantified by cell counting at days 3 and 6 post-inoculation (Figure 1). At an MOI of 5, UPEC-infected cells continue to proliferate between days 3 and 6 , although at a reduced rate, demonstrating that the described infection protocol is not cytotoxic. A similarly

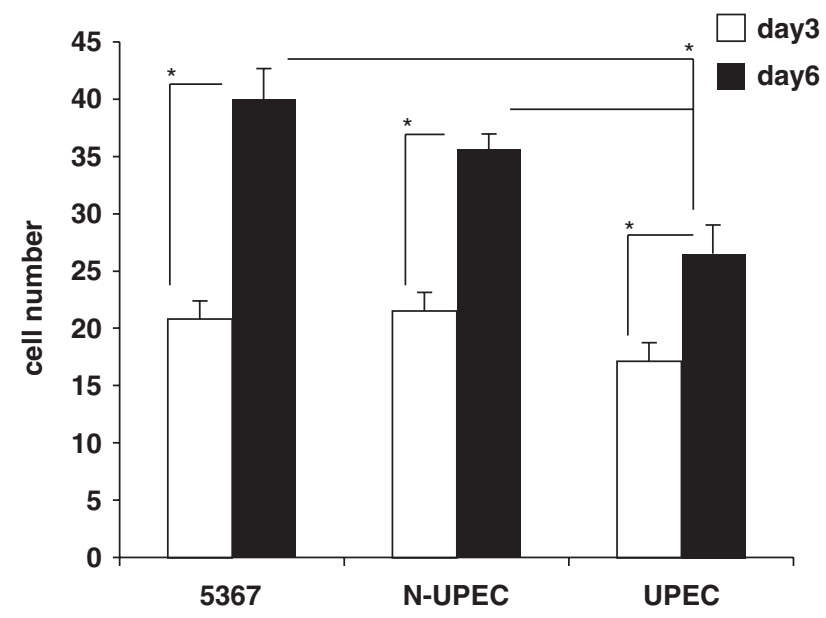

Figure 15637 cells inoculated with UPEC or N-UPEC continue to proliferate. In all, 5637 cells were inoculated as described in Materials and methods. Three and 6 days after inoculation, nuclei of DAPI-stained cells were counted. Values represent mean of $N=3 \pm$ s.e.m. Student's $t$-test was used to calculate $P$-values.

reduced proliferation rate was seen with N-UPEC inoculated cells.

One of the hallmarks of persistent infection is the presence of intracellular bacterial colonies. To demonstrate that our infection protocol leads to persistence of intracellular bacteria colonies, we stained inoculated cells with anti-E. coli antibodies. N-UPEC inoculated and non-inoculated cells were used as controls. UPEC-infected, N-UPEC inoculated and non-inoculated 5637 cells were cultured post-inoculation for 1-6 days, stained with anti-E. coli antibodies and analyzed by confocal microscopy. Only infection with UPEC resulted in internalization of bacteria, no bacteria were present inside N-UPEC inoculated or non-inoculated 5637 cells. Image analysis revealed that, although the percentage of infected cells decreased over time (Figure 2a), the number of bacteria per cell significantly increased between days 1 and 2 postinfection and remained constant between days 2 and 5 (Figure 2b), suggesting that UPEC not only persisted but also proliferated inside uroepithelial cells (Figure $2 \mathrm{a}$ and $\mathrm{b}$ ).

\section{UPEC Infection Stimulates DNA Methyltransferase Activity}

Since DNMT activity is responsible for increases in DNA methylation, we postulated that changes in DNMT activity would be an indicator for DNA methylation changes. Since cell division is usually associated with methylation of $\mathrm{CpG}$ islands by DNMTs, ${ }^{38}$ we increased the post-inoculation period to 6 days to allow uroepithelial cell proliferation and DNA methylation to occur. Endogenous total DNMT activity of UPEC and N-UPEC inoculated 5637 cells was analyzed using nuclear extracts. Nuclear extracts of HeLa cells and UPEC were used as positive and negative control, respectively. Infection of 5637 cells with UPEC increased DNMT activity 12.5 -fold compared with non-inoculated cells $(P=0.002)$, 

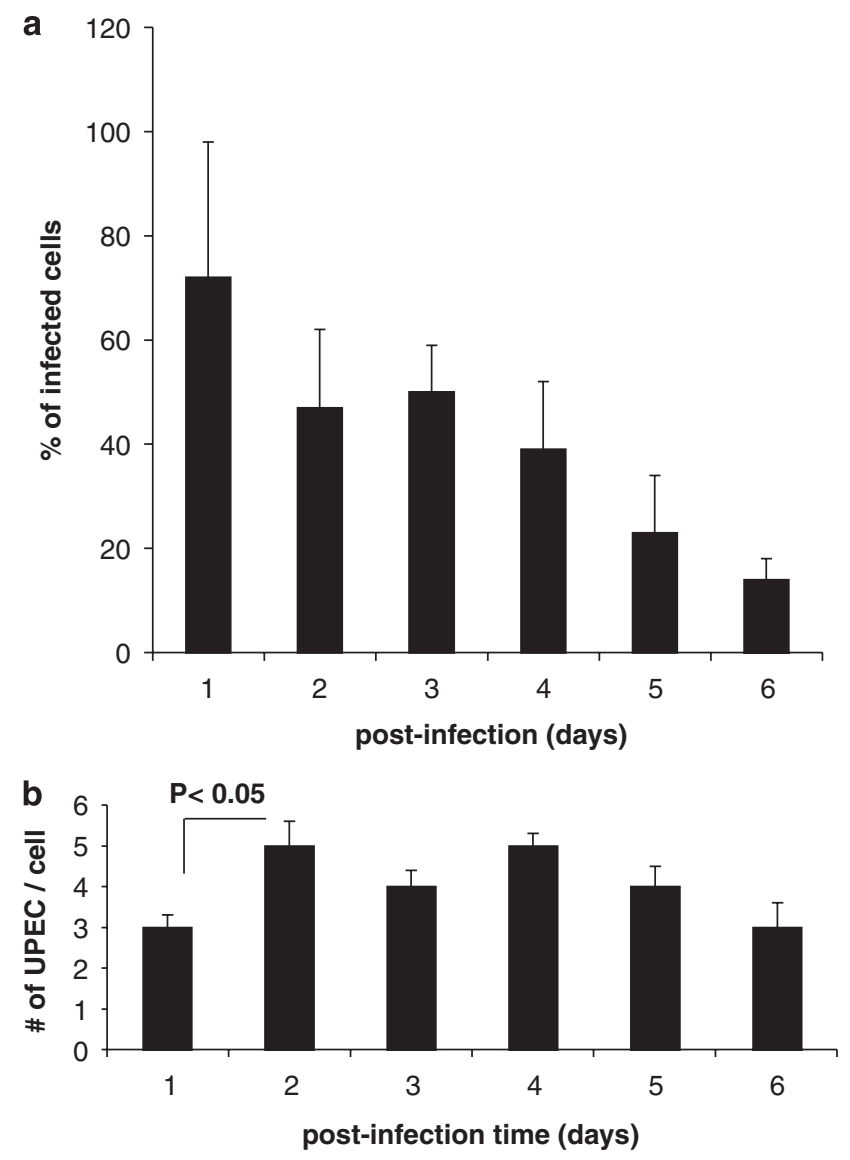

Figure 25637 cells internalize UPEC but not N-UPEC. UPEC are internalized by 5637 cells and persist intracellularly for the entire experimental period of up to 6 days. In all, 5637 cells were inoculated with UPEC or N-UPEC as described in Materials and methods. One to 6 days post-inoculation, cells were stained with antibodies against $E$. coli. Internalized bacteria and cells were counted using confocal microscopy images. (a) Graph depicts number of infected cells over time. (b) Graph depicts number of internalized $E$. coli divided by total number of cells. Values represent mean of $N=5 \pm$ s.e.m. Student's $t$-test was used to calculate $P$-values.

and 250-fold compared with N-UPEC inoculated cells $(P=0.001)$ (Figure 3a), suggesting that uroepithelial cell DNA might get specifically modified in UPEC-infected cells. Interestingly, uroepithelial cell inoculation with N-UPEC decreased total DNMT activity compared with non-inoculated control cells, suggesting that the presence of N-UPEC has an inhibitory effect on DNA methylation that is independent of $E$. coli internalization.

\section{UPEC Infection Stimulates DNMT1 Expression}

Since DNMT1 is recognized as the 'maintenance DNMT' in somatic cells, we investigated whether increased DNMT1 mRNA expression correlates with the observed high DNMT activity in UPEC-infected cells. In all, 5637 cells were infected with UPEC for $2 \mathrm{~h}$ followed by 6 days of postinoculation incubation. Real-time PCR revealed a 6-fold increase in DNMT1 mRNA expression compared with
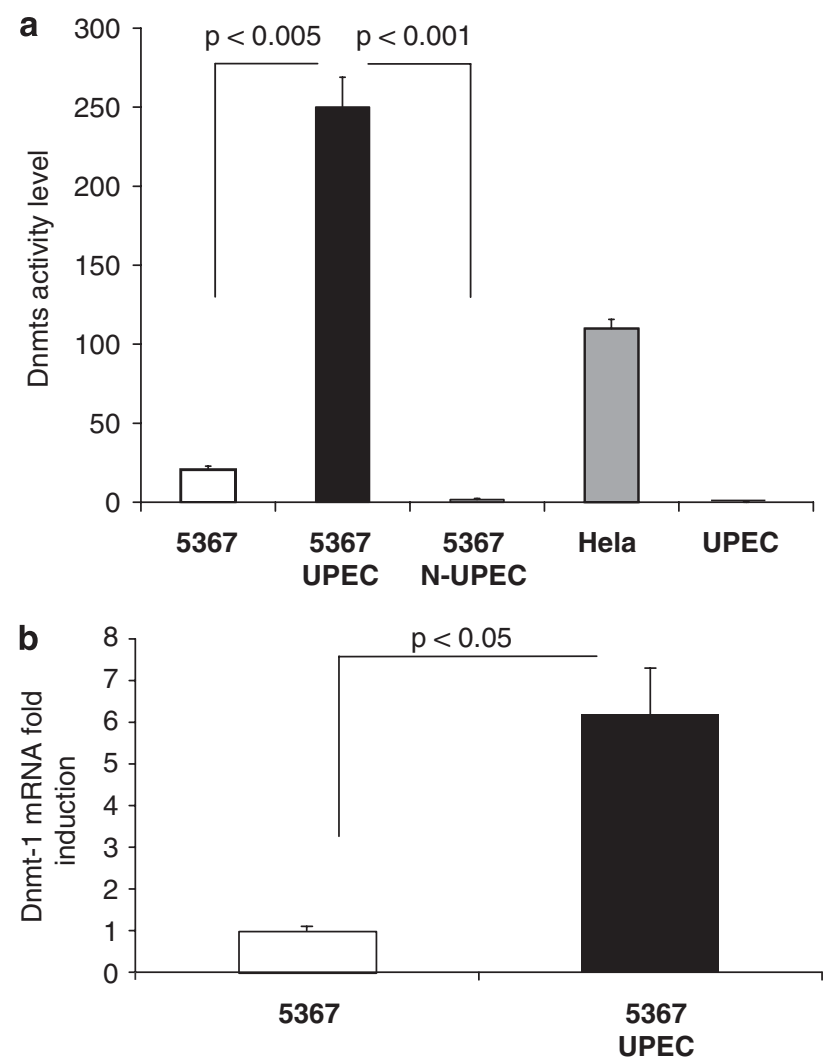

Figure 3 UPEC infection of 5637 cells results in upregulation of endogenous DNA methyltransferase activity and DNMT1 expression. (a) In all, 5637 cells were inoculated with either UPEC or N-UPEC as described in Materials and methods. DNMT activity was measured 6 days post-inoculation and was increased 12.5-fold compared with non-inoculated cells, and 250-fold compared with N-UPEC inoculated cells. HeLa cell nuclear extract was used as a positive and UPEC nuclear extracts as a negative control. (b) Infection of 5637 cells with UPEC resulted in 6-fold increase in DNMT1 mRNA expression compared with non-inoculated cells. DNMT1 and GAPDH expression was analyzed by real-time PCR. Quantitative analysis was performed by normalizing the DNMT1 values to matched GAPDH measurements and then calculating the ratio inoculated cells/non-inoculated (control) cells. Values represent mean of $N=3 \pm$ s.e.m. Student's $t$-test was used to calculate $P$-values.

non-inoculated uroepithelial cells $(P=0.04)$ (Figure $3 b$ ), suggesting that the high DNMT activity seen in UPECinfected cells is at least partly due to an increase in DNMT1 mRNA expression.

The increase in host DNMT1 expression could be either directly mediated by the internalized bacteria themselves, or an indirect consequence of factors that are released by infected cells. To gain insight into the mechanism that causes DNMT1 upregulation, we asked whether the E. coli infectioninduced DNMT1 expression is restricted to infected cells or is also manifested in non-infected cells that are co-cultured with infected cells. DNMT1 expression was analyzed on a single-cell level 6 days post-inoculation using immunofluorescent staining of DNMT1 followed by confocal microscopy and image analysis. Infected cells were identified 
by staining with an antibody against $E$. coli proteins. As a ubiquitous protein, DNMT1 localized to the nucleus in UPEC and N-UPEC inoculated cells and in non-inoculated control cells. However, 6 days post-inoculation, DNMT1 staining was 1.6-fold increased in UPEC-infected cells compared with non-inoculated cells $\left(P=3 \times 10^{-8}\right)$ and about 1.4-fold increased compared with N-UPEC inoculated cells $\left(P=7.3 \times 10^{-5}\right)$ (Figure $4 \mathrm{a}$ and $\mathrm{b}$ ). Furthermore, N-UPEC inoculation increased DNMT1 expression 1.2-fold compared with non-inoculated cells $(P=0.026)$, suggesting that mechanisms independent of bacteria internalization also have a role (Figure $4 \mathrm{a}$ and $\mathrm{b}$ ).

Analysis of DNMT1 levels of single cells within the UPECinfected cell population revealed that individual E. coli-containing cells express higher DNMT1 levels compared with individual cells that do not contain E. coli within the UPECinfected cell population (Figure 4c). Nevertheless, DNMT1 levels in individual cells of the UPEC-infected population remained significantly higher compared with N-UPEC inoculated or non-inoculated cells. These results support paracrine as well as autocrine mechanisms for bacterial infection-induced DNMT1 upregulation. A similar infectioninduced increase in DNMT1 levels was observed in two additional uroepithelia cell lines, J82 and HT-1197, suggesting that the effect of UPEC infection on DNMT1 expression is not restricted to one specific cell line but is a more prevalent phenomenon (Figure $4 \mathrm{~d}$ and e).

\section{UPEC Infection Downregulates CDKN2A and MGMT Expression}

Gene-specific DNA hypermethylation is generally associated with downregulation of gene expression. Since elevated DNMT1 mRNA transcription and DNMT activity in UPEC vs N-UPEC inoculated uroepithelial cells is suggestive of alterations in DNA methylation, we sought downregulation of expression of genes that had previously been implicated in infectious pathogenesis in bladder and gastric cancers. Expression of these genes in UPEC and N-UPEC inoculated 5637 cells was compared using real-time PCR. We initially focused our investigation on genes that are methylated in response to Schistosoma infection of the bladder and H. pylori infection of the gastric mucosa, such as CDKN2A, MLH1 and MGMT, as well as other candidate genes such as $C D H 1$ (E-cadherin, cell-cell adhesion) and DAPK1 (death-associated protein kinase 1, apoptosis). Infection of 5637 cells with UPEC resulted in a 3.3-fold decrease in CDKN2A (p16INK4A) mRNA expression compared with non-inoculated $(P=0.007)$ or N-UPEC inoculated cells $(P=0.01) \quad$ (Figure 5a). Moreover, infection with UPEC significantly downregulated MGMT mRNA expression compared with non-inoculated cells or N-UPEC inoculated cells (Figure 5b). Interestingly, MGMT mRNA expression was increased by N-UPEC, concordant with the decrease in DNMT activity observed above, suggesting that it is pathogenic ( $v s$ non-pathogenic) bacteria that are specifically associated with gene methylation and expression downregulation. In contrast, inoculation with either UPEC or N-UPEC did not decrease mRNA expression for CDH1, MLH1, DAPK1 and TLR4 (Table 2), suggesting that only specific genes are affected by $E$. coli infection.

Inoculation of 5637 cells with UPEC or N-UPEC resulted in 2.3- and 1.87-fold increase in IL8 mRNA expression, respectively (Table 2), suggesting that some pro-inflammatory factors may be upregulated in response to in vitro uroepithelial cell infection, and are not specifically downregulated through UPEC-induced DNA methylation.

\section{UPEC Infection of Uroepithelial Cells Induces CpG Methylation in Exon 1 of CDKN2A}

Since UPEC infection induces upregulation of DNMT activity and relative silencing of CDKN2A (p16INK4A) and $M G M T$ mRNA expression, we asked whether DNA hypermethylation may be occurring in candidate $\mathrm{CpG}$ islands in these genes. DNA methylation was assessed by pyrosequencing. CDKN2A contains three $\mathrm{CpG}$ islands in the promoter region and one island in exon 1 (Figure 6a). We quantified DNA methylation of $29 \mathrm{CpGs}$ flanking the translation start site in exon 1 of the CDKN2A (p16INK4A) gene (Figure 6a and $b$ ).

Methylation of CpG sites located 11 to $98 \mathrm{nt}$ downstream of the translation start site in exon 1 was significantly increased in response to UPEC infection (Figure 7). Methylation of CpG 20 was most significantly affected. Furthermore, we quantified DNA methylation of $8 \mathrm{CpGs}$ located 14 nucleotides downstream of the MGMT transcription start site. Although infection with UPEC led to a decrease in MGMT transcription, the methylation status of the MGMT CpG positions assessed remained unchanged (data not shown).

\section{DISCUSSION}

The goal of this study was to demonstrate a proof of principle that infection of host uroepithelial cells, the first cell of contact in UTI, with UPEC can provoke an epigenetic response in host cell DNA. DNA hypermethylation is a relatively stable epigenetic modification that leads to changes in gene expression. DNA methylation is being tested as potential diagnostic marker for a variety of cancers such as prostate, colorectal, cervical, lung, bladder cancer and leukemias. ${ }^{39-43}$ Whereas hypermethylation of tumor suppressor genes is a widely accepted mechanism for tumor progression, DNA hypermethylation in response to UTI infection is currently an understudied field and no DNA methylation based biomarker for future UTI infection susceptibility exists. Our goal is to identify candidate regions as potential biomarkers for UTI proclivity, as well as reveal new candidate mechanisms associated with UTI pathogenesis. To study the process in vitro, we developed a model of persistent infection of human uroepithelial cell lines with UPEC. Although an in vitro infection model requires MOI optimization for each host cell line, variation of DNA methylation is 
a

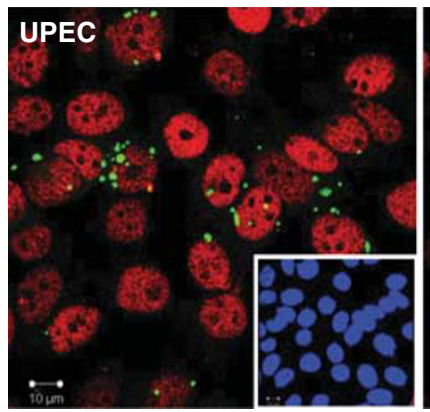

b

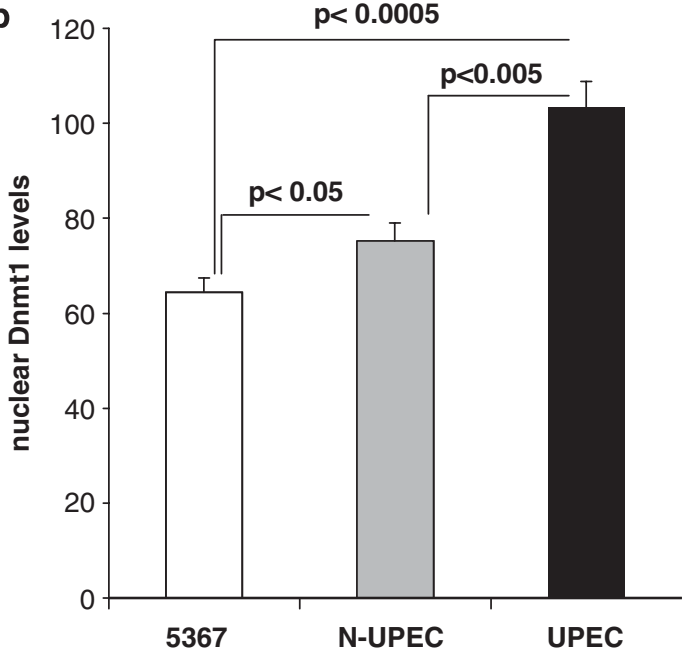

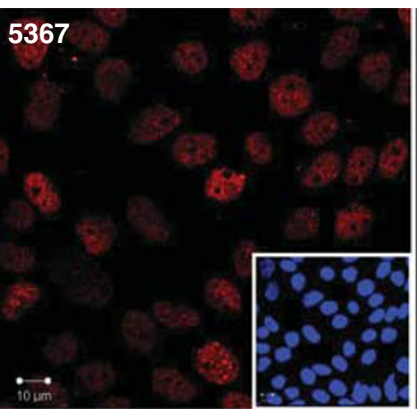

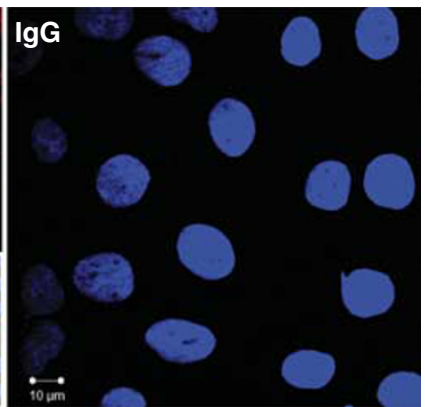

C

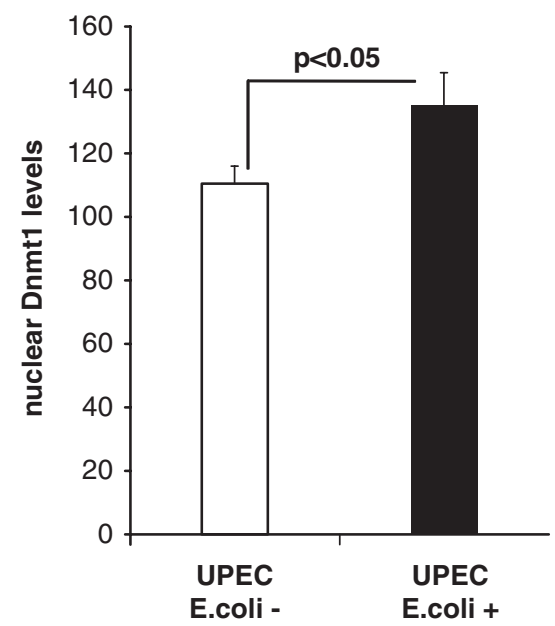

e

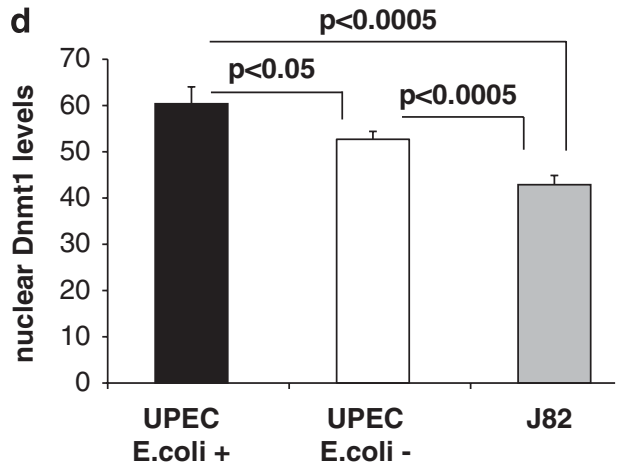

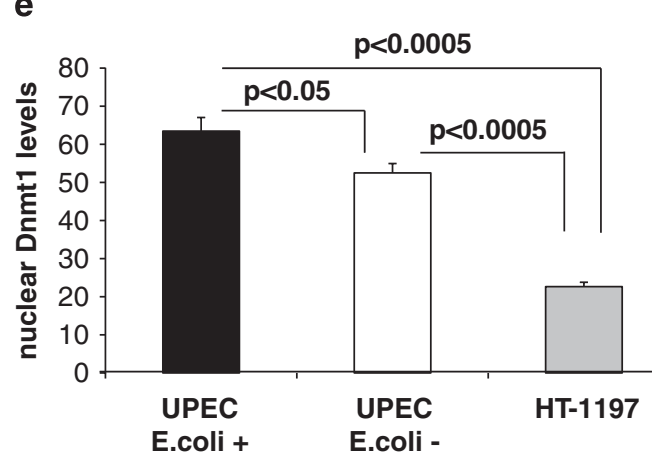

Figure 4 E. coli internalization is not required for DNMT1 upregulation. (a) UPEC-infected 5637 cells have increased nuclear DNMT1 levels compared with N-UPEC and non-inoculated cells. In all, 5637 cells were inoculated with UPEC or N-UPEC as described in Materials and methods. Six days post-inoculation, cells were double-stained with DNMT1 and E. coli-specific Ab. (b) Nuclear Dnmt1 levels were quantified by image analysis of confocal microscopy images. The graph compares DNMT1 levels of non-inoculated, N-UPEC and UPEC inoculated 5637 cells. (c) Nuclear DNMT1 levels of UPEC-infected cells that are void of internalized E. coli are higher compared with non-inoculated cells but slightly lower compared with $E$. coli-positive cells. Infected cells were identified by positive staining with E. coli-specific antibody. (d) Graph compares nuclear DNMT1 levels of E. coli-positive, E. coli-negative and non-inoculated J82 cells. (e) Graph compares nuclear DNMT1 levels of E. coli-positive, E. coli-negative and non-inoculated HT-1197 cells. Values represent mean of $N=3 \pm$ s.e.m. Student's $t$-test was used to calculate $P$-values.

reduced in an immortalized cell line compared with primary cells and patient samples, thereby increasing experimental reproducibility and allowing identification of relatively small methylation changes that may be missed in other experimental settings. Although an in vitro infection model has the disadvantage that cells of the immune system are absent, it allows efficient and reproducible analysis of infection-induced epigenetic consequences. Moreover, it allows analysis of infection-induced epigenetic consequences in the first cell of bacterial contact without the presence of 

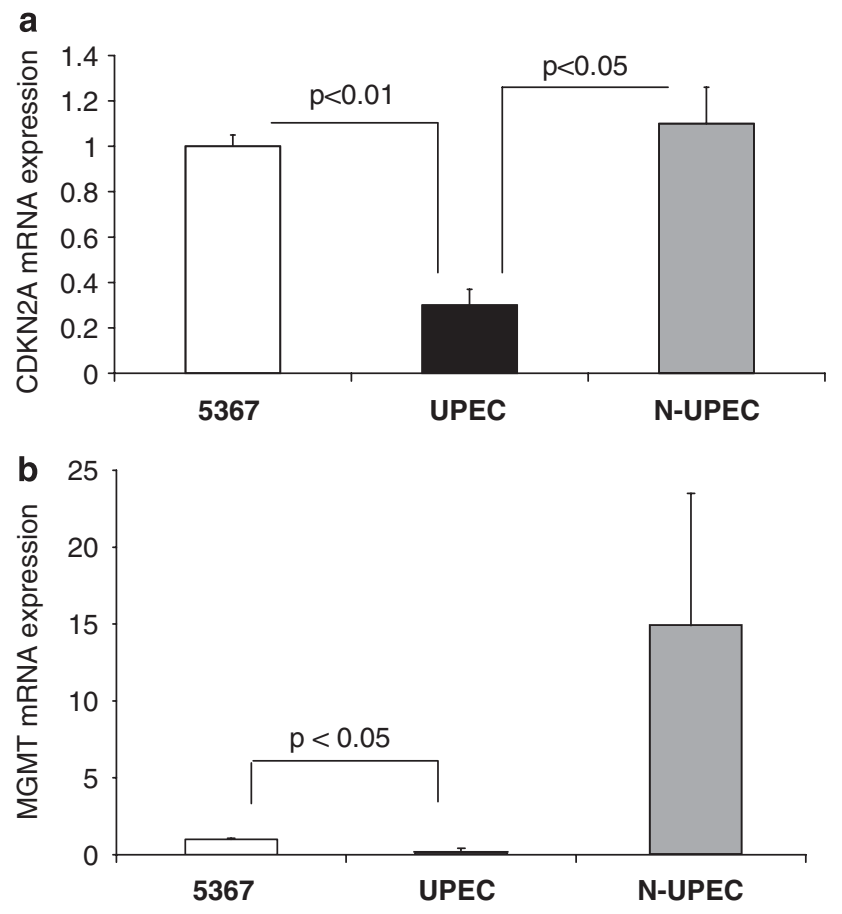

Figure 5 UPEC infection of 5637 cells results in downregulation of CDKN2A and MGMT. Infection of 5637 cells with UPEC resulted in 3.3-fold decrease of CDKN2A mRNA (a) and a 9.1-fold decrease of MGMT mRNA expression (b) compared with non-inoculated cells. CDKN2A, MGMT and $G A P D H$ expression was analyzed by real-time PCR. Quantitative analysis was performed by normalizing the CDKN2A or MGMT values to the corresponding GAPDH values and then calculating the ratio inoculated cells/non-inoculated (control) cells. Values represent mean of $N=3 \pm$ s.e.m. Student's $t$-test was used to calculate $P$-values.

Table 2 Infection of 5637 cells with FimH [+] E. coli results in mRNA downregulation of CDKN2A and MGMT compared to control cells or to FimH [-] E. coli-infected cells

\begin{tabular}{lccccc}
\hline $\begin{array}{l}\text { Gene } \\
\text { name }\end{array}$ & $\begin{array}{c}\text { Control } \\
5637 \text { cells }\end{array}$ & $\begin{array}{c}\text { FimH [+] } \\
\text { E. coli }\end{array}$ & $\begin{array}{c}\text { FimH [-] } \\
\text { E. coli }\end{array}$ & $* P$-value & **P-value \\
\hline CDKN2A/p16 & 1 & $\mathbf{0 . 3}$ & $\mathbf{1 . 1}$ & $\mathbf{0 . 0 0 7}$ & $\mathbf{0 . 0 4}$ \\
CDH1 & 1 & 1.7 & 1.5 & 0.16 & 0.21 \\
MLH1 & 1 & 1.2 & 0.9 & 0.17 & 0.55 \\
MGMT & 1 & $\mathbf{0 . 1 1}$ & 15 & $\mathbf{0 . 0 3}$ & 0.33 \\
DAPK1 & 1 & 1.4 & 1.4 & 0.19 & 0.675 \\
IL8 & 1 & 2.3 & 1.87 & 0.09 & 0.56 \\
TLR4 & 1 & 1 & 1.4 & 0.875 & 0.39
\end{tabular}

${ }^{*} P<0.05$ for FimH [+] E. coli-infected cells compared with control cells. ${ }^{*} P$-value for FimH [+] E. coli-infected cells compared with FimH [-] E. coliinfected cells.

Bold numbers indicate significant differences compared with control cells. Results were significant if $P<0.05 . N=3$.

effects on downstream cell types in the infection cascade on a single-cell level under controlled inoculation and timing conditions.
It is difficult to precisely relate the concepts of acute and persistent infection in a clinical sense to this in vitro model. Acute infection implies the early phases of bacterial exposure to host cells. Persistent infection refers to the host cellbacterial relationship in the days following initial bacterial exposure. Clinically, persistent infection can also imply how bacteria persist following antibiotic treatment-a feature that is best studied in patients. In our model, uroepithelial cells undergo some form of both of these phases: some host cells undoubtedly apoptose in vitro. We have been able to establish MOI conditions in vitro, however, that demonstrate that host cells also proliferate, as they do during clinical infection. ${ }^{44}$ Importantly, our assessments of epigenetic responses begin several days following inoculation to allow for bacterial internalization, which characterizes clinical persistence of infection. In this sense, the model is being studied for responses to persistent infection, as the clinical persistent infection phase is characterized by the presence of intracellular bacteria colonies. ${ }^{37}$ These colonies escape the immune system, and clinically can become reactivated and cause recurrent UTI. The resemblance between our in vitro model and the actual in vivo situation is of course limited because of the absence of an immune system, and 3D organ structure.

We employed a candidate gene approach to compare DNA methylation between cells inoculated with uropathogenic/ UPEC and non-pathogenic/N-UPEC, or non-inoculated cells. We reasoned initially that infection pathobiology would most likely be influenced by genes that have a role in host cell/pathogen interactions, cell survival, and innate immunity and concentrated our efforts on genes previously demonstrated to be regulated by DNA methylation in other experimental settings.

Since DNA hypermethylation influences gene expression, we initially quantified expression of these candidate genes in infected and non-infected cells. Using this approach we identified two genes, CDKN2A, encoding p16INK4A, and $M G M T$ that were downregulated in response to infection with UPEC but not in response to inoculation with N-UPEC. In contrast, inoculation with either UPEC or N-UPEC did not decrease mRNA expression for CDH1, MLH1, DAPK1 and TLR4, although these genes are well known to be inactivated by DNA methylation. ${ }^{23}$ TLR4 is a receptor for the bacterial FimH adhesin and LPS and TLR4 activation governs UTI susceptibility via stimulation of the host immunity. ${ }^{13,45-49}$ For example, TLR4 activation in epithelial cells leads to chemokine and cytokine production. ${ }^{45,46,48}$ Interestingly, UTI clearance requires TLR4 expression by bladder uroepithelial and innate immune cells. Analysis of TLR4 polymorphism revealed that certain TLR4 genotypes correlate with UTI recurrence or persistent asymptomatic infection in adult women and children. ${ }^{50-52}$ Also, TLR4 mutant mice develop chronic asymptomatic infections. ${ }^{49}$ Although we did not observe changes in TLR4 gene expression in our in vitro UTI infection model, TLR4 may still be epigenetically altered by methylation or histone modification 
a
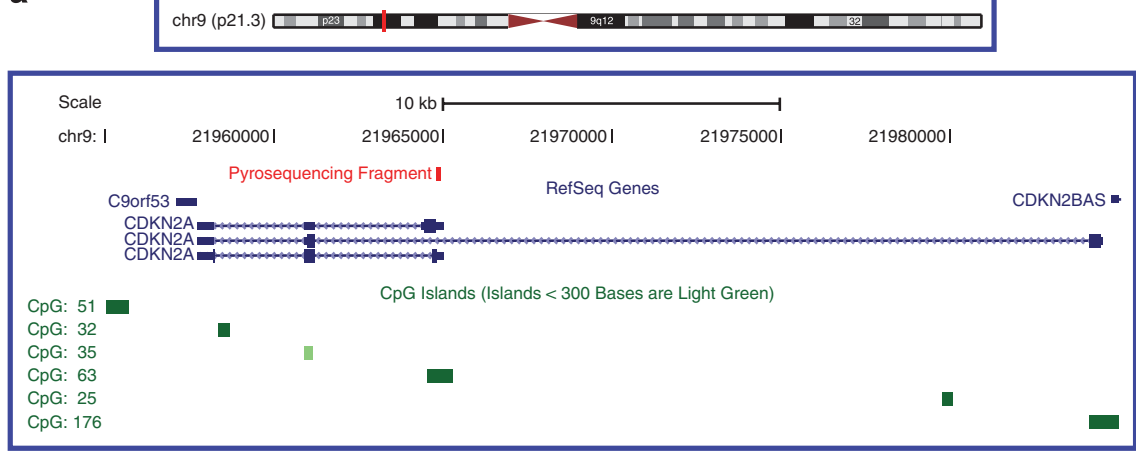

b gctgcttccggctggtgccccogggggagaCCCAACCTGGGGCGACTTCAGGGGTGCCA

CATTCGCTAAGTGCTCGGAGTTAATAGCACCTCCTCCGAGCACTCGCTCACG

GCGTCCCCTTGCCTGGAAAGATACCGCGGTCCCTCCAGAGGATTTGAGGGA

CAGGGTCGGAGGGGGCTCTTCCGCCAGCACCGGAGGAAGAAAGAGGAGG

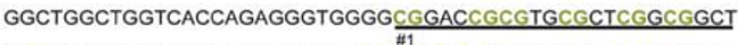
GCGGAGAGGGGGAGAGCAGGCAGCGGGCGGCGGGGAGCAGCATGGAGC $\frac{\text { CGGCGGCGGGGAGCAGCATGGAGCCTTCGGCTGACTGGCTGGCCACGGC }}{\# 13}$ CGCGGCCCGGGGTCGGGTAGAGGAGGTGCGGGCGCTGCTGGAGGCGGG

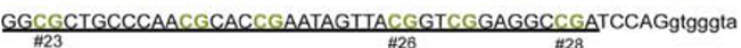
gagggtctgca

Figure 6 Diagram of CDKN2A CpG islands and sequence analyzed by pyrosequencing. (a) CpG islands are shown as green boxes, region analyzed by pyrosequencing is shown in red. (b) Translation start codon is shown in red, sequence analyzed is underlined and CpG dinucleotides are shown as green letters.

in response to UTI in vivo. In addition, future studies that incorporate UTI-induced cell-cell interactions that are present in vivo, but absent in our in vitro system, may yet reveal epigenetic involvement of TLR4 in UPEC infection.

CDKN2A (p16INK4A) is a well-known tumor suppressor gene and is involved in cell cycle regulation. ${ }^{53} \mathrm{P} 16 \mathrm{INK} 4 \mathrm{~A}$ is a G1-specific cell cycle inhibitor and reduces cell proliferation by inhibiting phosphorylation of the $\mathrm{Rb}$ protein by cyclindependent kinases CDK4 and CDK6. ${ }^{53}$ Although P16INK4A deletion has an undisputed role in transitional bladder carcinoma progression, P16INK4A DNA methylation is highly variable between individual bladder carcinoma patients and the contribution of epigenetic factors to bladder carcinoma risk is still controversial. ${ }^{54,55}$ UTI-induced CDKN2A methylation could provide a mechanism for the observed correlation between recurrent UTI and bladder carcinoma risk. ${ }^{56-58}$

After infection, bladder uroepithelial cells undergo apoptosis that results in shedding of infected cells and intracellular pathogens, thereby reducing the overall pathogen load. Cell proliferation of basal cells increases and is followed by cell differentiation to replace lost uroepithelial cells. ${ }^{59,60}$ UPEC-induced downregulation of CDKN2A may increase uroepithelial cell proliferation, thereby counteracting infection stimulated host uroepithelial cell apoptosis. This may increase pathogen persistence, ultimately increasing UTI recurrence risk.
MGMT is a DNA repair key enzyme that removes alkyl groups from the $\mathrm{O}^{6}$ position of guanine nucleotides and thereby prevents base transitions that would otherwise result in inactivation of important tumor suppressor genes, such as p53 or activation of oncogenes. ${ }^{61}$ It can be speculated that downregulation of MGMT in infected uroepithelial cells induces a mutator phenotype; accumulation of mutations could possibly increase pathogen persistence or uroepithelial cell re-infection, thereby increasing UTI recurrence risk. Although the observed effects on gene expression are rather moderate, they are likely additive and have the potential to increase UTI infection risk at multiple levels.

Interestingly, inoculation with N-UPEC upregulated MGMT expression, compared with control or UPEC-infected cells. This higher MGMT expression in N-UPEC inoculated cells correlated very well with reduced DNMT activity seen in N-UPEC inoculated cells, suggesting that inoculation with N-UPEC might inhibit MGMT DNA methylation. Therefore, N-UPEC may have potential for a probiotic effect and counteracting UPEC-induced epigenetic changes in the host genome. A probiotic effect may be one that has beneficial defensive characteristics in the face of pathogenic infection. The expression results may be the epigenetic reflection of such characteristics. Probiotic effects against UTI have already been described for Lactobacillus rhamnosus and L. fermentum. ${ }^{62,63}$ 

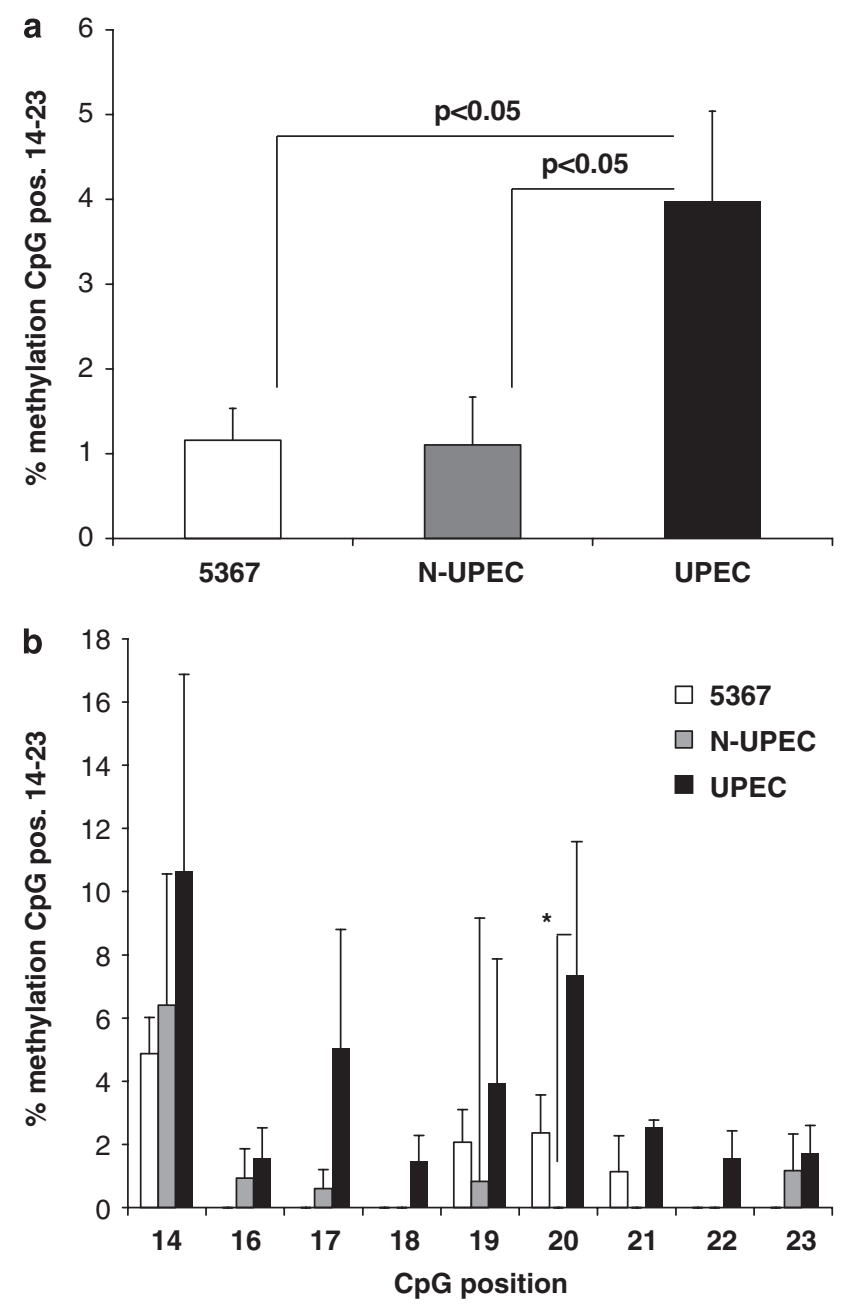

Figure 7 UPEC infection of 5637 cells results in hypermethylation of CDKN2A exon 1. In all, 5637 cells were inoculated with UPEC or N-UPEC as described in Materials and methods. Six days post-inoculation, methylation of individual CpG in the CDKN2A (p16INK4A) promoter region and exon 1 was quantified by pyrosequencing. (a) Graph depicts overall percentage methylation of $\mathrm{CpG}$ islands pos. 14-23. (b) Graph depicts percentage methylation of individual $\mathrm{CpG}$ dinucleotides in the same region as in (a). Values represent mean of $N=3 \pm$ s.e.m. ANOVA was used to calculate $P$-values.

Inoculation with both, UPEC or N-UPEC increased expression of IL8, an important player in the innate immune response. These result are consistent with the clinical situation, providing an exciting validation to our in vitro model. Several studies reported suppression of cytokine production in response to UPEC in vitro infection..$^{35,64,65}$ This discrepancy with our results is likely due to differences in post-infection incubation time and is therefore reflecting differences between acute and persistent phase of infection.

Both, CDKN2A (p16INK4A) and MGMT are regulated by hypermethylation of $\mathrm{CpG}$ islands in other pathological situations. Interestingly, UPEC infection increases methylation of the CDKN2A (p16INK4A) exon 1 CpG island 3.8-fold. In contrast, methylation of CpGs in the CDKN2A (p16INK4A) or MGMT promoter region was not changed. Hypermethylation of the CDKN2A (p16INK4A) promoter region correlates with tumor progression and is also a consequence of H. pylori infection. ${ }^{66}$ Therefore, CDKN2A (p16INK4A) DNA methylation patterns (exon $1 v s$ promoter) may depend on the specific factor inducing DNA methylation (E. coli vs H. pylori infection).

The observed methylation difference is based on analysis of a heterogeneous cell population consisting of infected and non-infected cells; fold changes in only infected single cells are likely much higher. Furthermore, this study does not represent a comprehensive genome-wide analysis of DNA methylation. Therefore, other genes are likely similarly affected. The final effect of infection-induced hypermethylation will be determined by the combination of all these modifications.

DNMT, the enzyme responsible for de novo DNA methylation, is upregulated in response to pathogenic infection in our model and increased DNMT activity correlates with augmented DNMT1 expression. We cannot rule out stimulation of DNMT activity by extra-transcriptional factors, as is the case for Epstein-Barr virus latent membrane protein 1 induced DNMT1 activity ${ }^{29}$ and enhanced expression of other members of the DNMT family (DNMT3A, DNMT3B) as mechanism for the observed DNMT activity increase.

Since our experiments did not reveal any changes in MGMT promotor CpG methylation, expression of this gene may be more specifically regulated by cell signaling, rather than epigenetic alteration in response to infection. For example, infection has been previously shown to increase interferon release, which potentially induces downregulation of CDKN2A (p16INK4A) and MGMT expression. Immunocytochemistry in combination with image analysis revealed that infection with UPEC not only affects DNMT1 expression of cells that contain bacteria but also neighboring cells that either escaped infection or cleared the bacteria. Nevertheless, DNMT1 levels were highest in cells that harbor E. coli. The observed gene regulation therefore likely results from a combination of downstream effects induced by diffusible factors and direct host cell responses to bacteria adhesion and uptake. Although previous studies found a correlation between DNMT levels and DNA methylation in cancer, more recent studies demonstrated that DNA methylation is not strictly influenced by DNMT levels but also by factors that recruit DNMTs to promoter regions. Consequently, the observed CDKN2A (p16INK4A) methylation increase can be a direct result of increased DNMT activity or increased DNMT recruitment. Since the methylation increase is specific for CDKN2A (p16INK4A), without affecting MGMT or overall DNA methylation (data not shown), DNMT recruitment likely has a role in UTI-induced CDKN2A methylation. In summary, this study provides a proof-of-principle that human uroepithelial cells undergo epigenetic modification following pathogenic E. coli infection. UPEC infection of uroepithelial cells results in hypermethylation of CDKN2A 
(p16INK4A) exon 1, which correlates with downregulation of CDKN2A (p16INK4A) expression. Although MGMT expression is downregulated in UPEC-infected cells, DNA methylation of this gene was not affected. E-cadherin (CDH1), MLH1 and DAPK and TLR4 were not specifically downregulated by UPEC infection in our model. Furthermore, these results suggest that infection triggers pathogen-specific alterations of the host cell epigenome, and not general microbe-induced gene downregulation.

Although this study is not a comprehensive analysis of DNA methylation and gene expression in UPEC-infected cells, it supports the search for epigenetically regulated biomarker genes for UTI recurrence. In future studies, CDKN2A (p16INK4A) methylation will be tested in DNA isolated from shed human urinary uroepithelial cells from patients followed longitudinally for UTI recurrence.

\section{ACKNOWLEDGEMENTS}

We thank Drs Scott Hultgren and Swaine Chen for generously providing the bacteria strains for this study. This study was supported by grants from the Hospital for Sick Children New Ideas funding (DJB) and Physician Services Incorporated (DJB and CT).

\section{DISCLOSURE/CONFLICT OF INTEREST}

The authors declare no conflict of interest.

1. Litwin MS, Saigal CS, Beerbohm EM. The burden of urologic diseases in America. J Urol 2005;173:1065-1066.

2. Litwin MS, Saigal CS, Yano EM, et al. Urologic diseases in America Project: analytical methods and principal findings. J Urol 2005;173: 933-937.

3. Kantor AF, Hartge $P$, Hoover RN, et al. Urinary tract infection and risk of bladder cancer. Am J Epidemiol 1984;119:510-515.

4. Jiang $X$, Castelao JE, Groshen S, et al. Urinary tract infections and reduced risk of bladder cancer in Los Angeles. Br J Cancer 2009;100:834-839.

5. Hooton TM, Stamm WE. Diagnosis and treatment of uncomplicated urinary tract infection. Infect Dis Clin North Am 1997;11:551-581.

6. Zhou G, Mo WJ, Sebbel P, et al. Uroplakin la is the urothelial receptor for uropathogenic Escherichia coli: evidence from in vitro FimH binding. J Cell Sci 2001;114(Part 22):4095-4103.

7. Mulvey MA, Lopez-Boado YS, Wilson CL, et al. Induction and evasion of host defenses by type 1-piliated uropathogenic Escherichia coli. Science 1998:282:1494-1497.

8. Mysorekar IU, Hultgren SJ. Mechanisms of uropathogenic Escherichia coli persistence and eradication from the urinary tract. Proc Natl Acad Sci USA 2006;103:14170-14175.

9. Martinez JJ, Mulvey MA, Schilling JD, et al. Type 1 pilus-mediated bacterial invasion of bladder epithelial cells. EMBO J 2000;19:2803-2812.

10. Mulvey MA, Schilling JD, Martinez JJ, et al. Bad bugs and beleaguered bladders: interplay between uropathogenic Escherichia coli and innate host defenses. Proc Natl Acad Sci USA 2000;97:8829-8835.

11. Hannan TJ, Mysorekar IU, Hung CS, et al. Early severe inflammatory responses to uropathogenic E. coli predispose to chronic and recurrent urinary tract infection. PLoS Pathog 2010;6.

12. Weichhart T, Haidinger $\mathrm{M}, \mathrm{Horl} \mathrm{WH}$, et al. Current concepts of molecular defence mechanisms operative during urinary tract infection. Eur J Clin Invest 2008;38(Suppl 2):29-38.

13. Song J, Abraham SN. Innate and adaptive immune responses in the urinary tract. Eur J Clin Invest 2008;38(Suppl 2):21-28.

14. Stapleton A. Host factors in susceptibility to urinary tract infections. Adv Exp Med Biol 1999;462:351-358.

15. Finer $G$, Landau $D$. Pathogenesis of urinary tract infections with normal female anatomy. Lancet Infect Dis 2004;4:631-635.

16. Prandota J. Urinary tract diseases revealed after DTP vaccination in infants and young children: cytokine irregularities and down-regulation of cytochrome P-450 enzymes induced by the vaccine may uncover latent diseases in genetically predisposed subjects. Am J Ther 2004;11: 344-353.

17. Zhang D, Zhang G, Hayden MS, et al. A toll-like receptor that prevents infection by uropathogenic bacteria. Science 2004;303:1522-1526.

18. Poltorak A, He X, Smirnova l, et al. Defective LPS signaling in $\mathrm{C} 3 \mathrm{H} / \mathrm{HeJ}$ and C57BL/10ScCr mice: mutations in Tlr4 gene. Science 1998;282: 2085-2088.

19. Qureshi ST, Lariviere L, Leveque $\mathrm{G}$, et al. Endotoxin-tolerant mice have mutations in Toll-like receptor 4 (TIr4). J Exp Med 1999;189:615-625.

20. Lorenz E, Mira JP, Frees $\mathrm{KL}$, et al. Relevance of mutations in the TLR4 receptor in patients with gram-negative septic shock. Arch Intern Med 2002;162:1028-1032.

21. Schmitt C, Humeny A, Becker CM, et al. Polymorphisms of TLR4: rapid genotyping and reduced response to lipopolysaccharide of TLR4 mutant alleles. Clin Chem 2002;48:1661-1667.

22. Jaenisch R, Bird A. Epigenetic regulation of gene expression: how the genome integrates intrinsic and environmental signals. Nat Genet 2003;33Suppl:245-254.

23. Gutierrez $\mathrm{Ml}$, Siraj $\mathrm{AK}$, Khaled $\mathrm{H}$, et al. $\mathrm{CpG}$ island methylation in Schistosoma- and non-Schistosoma-associated bladder cancer. Mod Pathol 2004;17:1268-1274.

24. Miyazaki T, Murayama $Y$, Shinomura $Y$, et al. E-cadherin gene promoter hypermethylation in $\mathrm{H}$. pylori-induced enlarged fold gastritis. Helicobacter 2007;12:523-531.

25. Ushijima T, Nakajima T, Maekita T. DNA methylation as a marker for the past and future. J Gastroenterol 2006;41:401-407.

26. Yao $\mathrm{Y}$, Tao $\mathrm{H}$, Park $\mathrm{Dl}$, et al. Demonstration and characterization of mutations induced by Helicobacter pylori organisms in gastric epithelial cells. Helicobacter 2006;11:272-286.

27. Uozaki H, Fukayama M. Epstein-Barr virus and gastric carcinoma - viral carcinogenesis through epigenetic mechanisms. Int J Clin Exp Pathol 2008;1:198-216.

28. Burgers WA, Blanchon L, Pradhan S, et al. Viral oncoproteins target the DNA methyltransferases. Oncogene 2007;26:1650-1655.

29. Seo SY, Kim EO, Jang KL. Epstein-Barr virus latent membrane protein 1 suppresses the growth-inhibitory effect of retinoic acid by inhibiting retinoic acid receptor-beta2 expression via DNA methylation. Cancer Lett 2008;270:66-76.

30. Lee JO, Kwun HJ, Jung JK, et al. Hepatitis B virus $X$ protein represses E-cadherin expression via activation of DNA methyltransferase 1. Oncogene 2005;24:6617-6625.

31. Kang GH, Lee S, Kim JS, et al. Profile of aberrant CpG island methylation along the multistep pathway of gastric carcinogenesis. Lab Invest 2003;83:635-641.

32. To KF, Leung WK, Lee TL, et al. Promoter hypermethylation of tumorrelated genes in gastric intestinal metaplasia of patients with and without gastric cancer. Int J Cancer 2002;102:623-628.

33. Aquilina G, Bignami M. Mismatch repair in correction of replication errors and processing of DNA damage. J Cell Physiol 2001;187:145-154.

34. Soejima H, Zhao W, Mukai T. Epigenetic silencing of the MGMT gene in cancer. Biochem Cell Biol 2005;83:429-437.

35. Hunstad DA, Justice SS, Hung CS, et al. Suppression of bladder epithelial cytokine responses by uropathogenic Escherichia coli. Infect Immun 2005;73:3999-4006.

36. Reigstad CS, Hultgren SJ, Gordon Jl. Functional genomic studies of uropathogenic Escherichia coli and host urothelial cells when intracellular bacterial communities are assembled. J Biol Chem 2007; 282:21259-21267.

37. Rosen DA, Hooton TM, Stamm WE, et al. Detection of intracellular bacterial communities in human urinary tract infection. PLoS Med 2007:4:e329.

38. Velicescu M, Weisenberger DJ, Gonzales FA, et al. Cell division is required for de novo methylation of $\mathrm{CpG}$ islands in bladder cancer cells. Cancer Res 2002;62:2378-2384.

39. Goel A. DNA methylation-based fecal biomarkers for the noninvasive screening of Gl cancers. Future Oncol 2010;6:333-336.

40. Shivapurkar N, Gazdar AF. DNA methylation based biomarkers in non-invasive cancer screening. Curr Mol Med 2010;10:123-132.

41. Vlassov VV, Laktionov PP, Rykova EY. Circulating nucleic acids as a potential source for cancer biomarkers. Curr Mol Med 2010;10: 142-165.

42. Qureshi SA, Bashir MU, Yaqinuddin A. Utility of DNA methylation markers for diagnosing cancer. Int J Surg 2010;8:194-198. 
43. Dehan P, Kustermans G, Guenin S, et al. DNA methylation and cancer diagnosis: new methods and applications. Expert Rev Mol Diagn 2009; 9:651-657.

44. Mysorekar IU, Isaacson-Schmid M, Walker JN, et al. Bone morphogenetic protein 4 signaling regulates epithelial renewal in the urinary tract in response to uropathogenic infection. Cell Host Microbe 2009; 5:463-475.

45. Mian MF, Lauzon NM, Andrews DW, et al. FimH can directly activate human and murine natural killer cells via TLR4. Mol Ther 2010;18: 1379-1388.

46. Ashkar AA, Mossman KL, Coombes BK, et al. FimH adhesin of type 1 fimbriae is a potent inducer of innate antimicrobial responses which requires TLR4 and type 1 interferon signalling. PLoS Pathog 2008;4: e1000233.

47. Mossman $\mathrm{KL}$, Mian MF, Lauzon NM, et al. Cutting edge: FimH adhesin of type 1 fimbriae is a novel TLR4 ligand. J Immunol 2008;181: 6702-6706.

48. Schilling JD, Mulvey MA, Vincent $C D$, et al. Bacterial invasion augments epithelial cytokine responses to Escherichia coli through a lipopolysaccharide-dependent mechanism. J Immunol 2001;166:1148-1155.

49. Ragnarsdottir B, Fischer H, Godaly G, et al. TLR- and CXCR1-dependent innate immunity: insights into the genetics of urinary tract infections. Eur J Clin Invest 2008;38(Suppl 2):12-20.

50. Zaffanello M, Malerba G, Cataldi L, et al. Genetic risk for recurrent urinary tract infections in humans: a systematic review. J Biomed Biotechnol 2010;2010:321082.

51. Hawn TR, Scholes D, Li SS, et al. Toll-like receptor polymorphisms and susceptibility to urinary tract infections in adult women. PLoS One 2009;4:e5990.

52. Karoly E, Fekete A, Banki NF, et al. Heat shock protein 72 (HSPA1B) gene polymorphism and Toll-like receptor (TLR) 4 mutation are associated with increased risk of urinary tract infection in children. Pediatr Res 2007:61:371-374.

53. Matsuda Y. Molecular mechanism underlying the functional loss of cyclindependent kinase inhibitors p16 and p27 in hepatocellular carcinoma. World J Gastroenterol 2008;14:1734-1740.

54. Florl AR, Franke $\mathrm{KH}$, Niederacher $\mathrm{D}$, et al. DNA methylation and the mechanisms of CDKN2A inactivation in transitional cell carcinoma of the urinary bladder. Lab Invest 2000;80:1513-1522.
55. Chapman EJ, Harnden $P$, Chambers $P$, et al. Comprehensive analysis of CDKN2A status in microdissected urothelial cell carcinoma reveals potential haploinsufficiency, a high frequency of homozygous codeletion and associations with clinical phenotype. Clin Cancer Res 2005; 11:5740-5747.

56. Lin $\mathrm{HH}, \mathrm{Ke} \mathrm{HL}, \mathrm{Wu}$ WJ, et al. Hypermethylation of E-cadherin, p16, p14, and RASSF1A genes in pathologically normal urothelium predict bladder recurrence of bladder cancer after transurethral resection. Urol Oncol 2010; doi:10.1016/j.urolonc.2010.01.002 (in press).

57. Phe V, Cussenot $O$, Roupret $M$. Interest of methylated genes as biomarkers in urothelial cell carcinomas of the urinary tract. BJU Int 2009;104:896-901.

58. Jarmalaite $\mathrm{S}$, Jankevicius F, Kurgonaite K, et al. Promoter hypermethylation in tumour suppressor genes shows association with stage, grade and invasiveness of bladder cancer. Oncology 2008;75: 145-151.

59. Mysorekar IU, Mulvey MA, Hultgren SJ, et al. Molecular regulation of urothelial renewal and host defenses during infection with uropathogenic Escherichia coli. J Biol Chem 2002;277:7412-7419.

60. Justice SS, Hung C, Theriot JA, et al. Differentiation and developmental pathways of uropathogenic Escherichia coli in urinary tract pathogenesis. Proc Natl Acad Sci USA 2004;101:1333-1338.

61. Esteller M, Herman JG. Generating mutations but providing chemosensitivity: the role of O6-methylguanine DNA methyltransferase in human cancer. Oncogene 2004;23:1-8.

62. HoesI CE, Altwein JE. The probiotic approach: an alternative treatment option in urology. Eur Urol 2005;47:288-296.

63. Bruce AW, Reid G. Probiotics and the urologist. Can J Urol 2003;10: 1785-1789.

64. Billips BK, Schaeffer AJ, Klumpp DJ. Molecular basis of uropathogenic Escherichia coli evasion of the innate immune response in the bladder. Infect Immun 2008;76:3891-3900.

65. Billips BK, Forrestal SG, Rycyk MT, et al. Modulation of host innate immune response in the bladder by uropathogenic Escherichia coli. Infect Immun 2007;75:5353-5360.

66. Alves MK, Lima VP, Ferrasi AC, et al. CDKN2A promoter methylation is related to the tumor location and histological subtype and associated with Helicobacter pylori flaA(+) strains in gastric adenocarcinomas. APMIS 2010;118:297-307. 